\title{
Research progress of carbon based nanoenzyme and composites in antibacterial field
}

\author{
ChuanXin Zhao ${ }^{1}$, QiaMin Gu ${ }^{1}$, GaoYun Chen ${ }^{1}$, MengBin Yu ${ }^{1}$, Min Liu ${ }^{1 *}$ \\ ${ }^{1}$ Institute of NBC Defense, Beijing, 102205, China
}

\begin{abstract}
Due to the abuse of antibiotics, more and more bacteria are resistant to antibiotics. Non antibiotic nano antibacterial materials emerge as the times require. Carbon based nano enzyme is an efficient and environmentally friendly antibacterial material with certain antibacterial effect. It has simple structure and good compatibility. It can be combined with a variety of antibacterial substances to form composite antibacterial materials, expand the scope of antibacterial and improve the antibacterial ability. This paper summarizes the research progress of three kinds of carbon based nanoenzymes including carbon nanotubes, graphene, carbon quantum dots and their composites in the field of antibacterial.
\end{abstract}

\section{Introduction}

In nature, bacteria and viruses are everywhere. To effectively kill bacteria and inhibit the growth and reproduction of bacteria is an important topic closely related to human health, which has been widely concerned by the scientific community. Antibiotics can effectively kill bacteria, but with the extensive use and abuse of antibiotics, the drug resistance of bacteria is gradually enhanced, and even super drug-resistant bacteria are produced. Therefore, a kind of green and environmentally friendly antibacterial material that will not make bacteria resistant is widely expected.

Nano antibacterial material is a kind of material that uses nano technology to treat inorganic antibacterial agent, so that it has more extensive and excellent bactericidal ability. It can achieve long-term antibacterial effect through slow-release effect. Nano enzyme is a kind of nano material with enzyme like catalytic activity, which can catalyze the substrate of natural enzyme under physiological conditions and follow similar reaction kinetics. Its catalytic activity comes from its own special nanostructure, which has higher catalytic activity, better stability and lower cost than natural enzyme or other artificial enzyme. It has a certain potential to be widely used in the field of antibacterial.

At present, more than 50 kinds of nanoenzymes have been found, mainly including metal, metal oxide and carbon nanomaterials. Most of the nanoenzymes have the activity of oxidoreductase, which can produce a large number of free radicals by catalytic oxidation-reduction reaction.

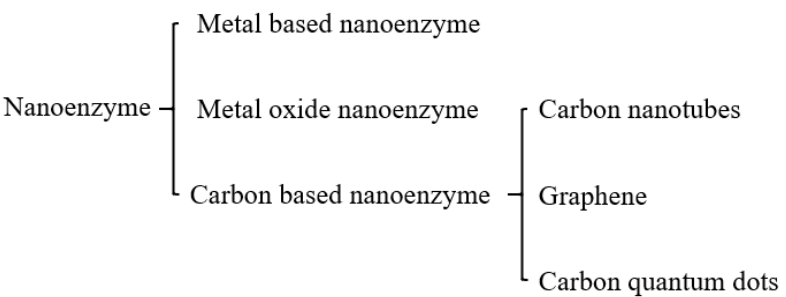

Figure 1 Classification of nanoenzymes

Among the above three kinds of nanoenzymes, carbon based nanoenzymes have good environmental friendliness, large specific surface area, excellent physical properties, as well as the adsorption and immobilization ability of molecules and nanoparticles, so they are an excellent choice as bactericidal materials and carriers. At present, carbon based nanoenzymes used in antibacterial field mainly include carbon nanotubes, graphene, carbon quantum dots and so on.

\section{Carbon nanotubes}

In 1991, Japanese scientist lijima ${ }^{1}$ discovered carbon nanotubes for the first time. This kind of carbon nanomaterials has high stability and large specific surface area. It has a tubular structure made of graphite sheets, in which carbon is bonded by sp 2 hybrid. According to the number of carbon atom layers in the wall of carbon nanotubes, they can be divided into single-walled carbon nanotubes (SWCNTs) and multi walled carbon nanotubes (MWCNTs). Due to its unique structure and excellent mechanical, electrical and chemical properties, carbon nanotubes show a broad application prospect, and attract the great attention of many scientists in materials, physics, electronics, chemistry and other fields. So it has been widely used.

In 2007, Kang et $\mathrm{al}^{2}$ reported for the first time that

\footnotetext{
*Corresponding author:979919775@qq.com
} 
single-walled carbon nanotubes have strong antibacterial activity. Direct contact between bacteria and single-walled carbon nanotubes can cause serious membrane damage and then cell inactivation. Therefore, single-walled carbon nanotubes have broad application prospects as new antibacterial materials.

The results show that the size, dispersion, concentration and buffer type of CNTs have some influence on their antibacterial activity.Yang et al. ${ }^{3}$ found that the length of SWCNTs affected their antibacterial activity. Under the same weight concentration of three different lengths of SWCNTs, the long SWCNTs showed stronger antibacterial activity than the short SWCNTs. The length of SWCNTs affects the aggregation of SWCNTs and bacterial cells, thus affecting the effect of direct interaction between cells and SWCNTs. Liu et al. ${ }^{4}$ found that SWCNT dispersion has higher antibacterial activity than SWCNT aggregates. The antibacterial activity of SWCNT dispersion against Gram-positive bacteria (Bacillus subtilis and Staphylococcus aureus) was also higher than that against Gram-negative bacteria (Escherichia coli and Pseudomonas aeruginosa). SWCNTs can be regarded as nanodarts moving in solution, attacking bacteria to degrade the integrity of bacterial cells and lead to cell death. The antibacterial activity of singlewalled carbon nanotubes can be further improved by dispersing single-walled carbon nanotubes, increasing the concentration of single-walled carbon nanotubes, and increasing the oscillation speed to enhance the physical penetration ability of single-walled carbon nanotubes to bacteria. Arias et al. ${ }^{5}$ found that SWNTs showed strong antibacterial activity only in deionized water or $0.9 \%$ $\mathrm{NaCl}$ solution, but not in PBS buffer and BHI broth. The antibacterial activity of SWNTs increased with the increase of concentration and treatment time.

In recent years, in order to enhance the antibacterial properties of carbon nanotubes, more and more experts and scholars focus on the preparation of composite antibacterial materials by loading some biochemical substances on carbon nanotubes. Silver with antibacterial properties has been widely concerned by the scientific community. Studies have shown that the antibacterial effect of Ag based composites is directly proportional to the degree of contact between $\mathrm{Ag}^{+}$and bacterial cells, because $\mathrm{Ag}^{+}$can effectively enter bacterial cells through bacterial cell membrane and prevent their growth.

Liu et al. ${ }^{6}$ successfully synthesized Ag / MWCNTs composite materials by ion beam assisted deposition technology, taking Gram-negative bacteria (E.coli) and Gram-positive bacteria (Staphylococcus aureus) as research objects. For two different bacteria, Ag / MWCNTs composite showed more obvious antibacterial effect than MWCNTs. It was found that the antibacterial effect of Ag / MWCNTs on Escherichia coli was better than that on Staphylococcus aureus, which may be because the cell wall of Gram-negative bacteria was thinner than that of Gram-positive bacteria, which made it easier for silver ions to penetrate the cell wall and enter the cells, leading to the death of bacteria. Zardini et al. ${ }^{7}$ found that lysine and arginine were used to functionalize multi walled carbon nanotubes (MWCNTs), and their antibacterial activities against Escherichia coli,
Staphylococcus and other bacteria were significantly improved. When MWCNTs Arg products were used against Gram-negative bacteria, Escherichia coli and Salmonella typhimurium, the enhancement of this activity was more obvious. This finding indicates that the positively charged amino acid cation group is very effective in destroying the bacterial membrane, which can destroy the integrity of the cell membrane and then kill the bacteria.

In 2012, alsan et al. ${ }^{8}$ successfully assembled LBL with SWNT containing polylysine (PLL) and polyglutamic acid (PGA), and observed a greater degree of SWNT incorporation in PL-PEG system. We found that SWNT / PLL / PGA membrane could significantly reduce the activity of Escherichia coli and Staphylococcus epidermidis ( $90 \%$ after 24 hours of Culture).

In 2014, Dong et al. ${ }^{9}$ found that the surface of MWCNTs adsorbed by nisin had strong anti adhesion and antibacterial effects on Bacillus anthracis. It reduced the attachment of spores and inhibited spore germination and biofilm formation. Compared with the control surface, the number of bound spores on the surface of MWCNT nisin decreased by 3.5 times, and the subsequent biofilm formation decreased by $94.6 \%$. The results showed that the surface of MWCNT nisin had the excellent physical properties of MWCNTs and nisin, and had potential application prospect in the preparation of anti adhesion and anti biofilm formation surface on the surface of anthrax spores.

\section{Graphene}

Graphene and its derivatives have strong antibacterial properties. The preparation of antibacterial nanocomposites using graphene and its derivatives has a wide application prospect.

In 2010 , Hu et al. ${ }^{10}$ studied the antibacterial ability of graphene oxide against E. coli, and found that E. coli liquid was placed in $20 \mu \mathrm{G} / \mathrm{ml}$ and $85 \mu$ The metabolic activity of E.coli decreased to $70 \%$ and $13 \%$ respectively after 2 hours in the graphene oxide dispersion $(\mathrm{g} / \mathrm{ml})$. It was also found that when the bacterial cells contacted with graphene derivatives, the cell membrane would be damaged, resulting in the loss of integrity of cell membrane and death.

Liu et al. ${ }^{11}$ compared the antibacterial activity of aqueous dispersion of graphite (GT), graphite oxide (GTO), graphene oxide (GO) and reduced graphene oxide (RGO) against Escherichia coli. The results showed that GO had the highest antibacterial activity at the same concentration, followed by RGO, GT and GTO. Its antibacterial activity is related to time and concentration. Most bacterial inactivation occurs in the first hour of culture, and cell mortality increases with the increase of substance concentration. They also found that the lateral size of graphene oxide had an effect on the antibacterial activity. The larger graphene oxide nanosheets showed stronger antibacterial activity, which could cause the death of most bacteria in only one hour, and also showed better antibacterial activity at a relatively low concentration.

Many researchers combine graphene and its 
derivatives with other nano materials, including silver nanoparticles and chitosan, to develop composite materials with stronger antibacterial ability, better stability and better biocompatibility.Liu et al. ${ }^{12}$ prepared GO-Ag nanocomposites by simple blending method. When the mass concentration of go AG is $80 \mu \mathrm{g} / \mathrm{ml}$, the bactericidal rate of GO-Ag composite to Escherichia coli is $99 \%$, which is much higher than that of go alone. This shows that go Ag composite can have high antibacterial performance at low concentration. The reason is that go Ag composite has large specific surface area, Ag nanoparticles are easy to precipitate on the surface of go, so go can be well adsorbed on the surface of go Therefore, the antibacterial activity is greatly improved.

Lim et al. ${ }^{13}$ measured the antibacterial activity of GO and $\mathrm{rGO}$ composite with chitosan respectively. The results showed that GO or rGO composite with chitosan did not affect its own antibacterial activity. Because GO and chitosan had synergistic antibacterial effect, the antibacterial activity of the composite was better than that of GO or rGO at the same concentration.

Sun et al. ${ }^{14}$ used graphene quantum dots to catalyze hydrogen peroxide, which can effectively kill gramnegative Escherichia coli and gram-positive Staphylococcus aureus. The required concentration of hydrogen peroxide is only $100 \mu \mathrm{mol} / \mathrm{L}$. This bactericidal ability can be used to prevent wound infection and promote wound healing. In addition, they also found ${ }^{15}$ that AuNPs with graphitic carbonnitride has high peroxidase activity and can also catalyze hydrogen peroxide at physiological level to kill drug-resistant Escherichia coli and Staphylococcus aureus, effectively prevent the formation of bacterial biofilm and accelerate the healing of infectious sores. More importantly, it can be injected into mice for the treatment of pulmonary drug-resistant bacteria infection, Its germicidal efficacy is similar to that of vancomycin. They ${ }^{16}$ further loaded gold nanoparticles onto mesoporous silica supported gold nanoparticles (MSN AuNPs) and found that the composite had oxidase and peroxidase activities, which synergistically enhanced the ability to produce free radicals, and could not only prevent the formation of Escherichia coli and Staphylococcus aureus biofilms, It can also destroy the formed biofilm.

On the basis of these works, the team ${ }^{17}$ also designed a chemical photothermal synergistic antibacterial system with the corresponding demand ability. The sandwich like graphene mesoporous silicon nanosheets (GS) were used as the carrier, and the prodrug ascorbic acid (ASA) was added into the system, Vancomycin (AA) was loaded into GS mesoporous and encapsulated with hyaluronic acid dopamine (HA). Then vancomycin modified iron oxide nanoenzyme was assembled on the outer surface. Vancomycin could target the antibody system to the surface of gram negative or positive bacteria, Once contacted with bacteria, Ha-Da can be degraded by hyaluronidase secreted by bacteria to release ascorbic acid. Iron oxide nanoenzyme catalyzes ascorbic acid to produce free radicals and finally kill bacteria. The photothermal effect of graphene can further improve the anti-bacterial effect. This system can effectively remove stubborn biofilm and prevent infection in vivo.

\section{Carbon quantum dots}

Carbon quantum dots (CQDS) have attracted much attention in the field of medicine and biology in recent years. Different from graphene based nanomaterials, CQDS are zero dimensional carbon nanomaterials with particle size less than $10 \mathrm{~nm}$, and have unique morphology, size, surface functional groups and physicochemical properties, which are easy to be doped with other elements (such as nitrogen, sulfur, chlorine, bromine, etc.) in the preparation process. CQDS have obvious advantages in water solubility and modifiability, and can easily prepare CQDS with different properties according to experimental needs ${ }^{18}$.

CDs have shown great potential in various antibacterial applications, which can eliminate bacterial infection and have relatively low toxic and side effects on mammalian cells and tissues. It is a complex process to study the antibacterial mechanism of CDs. Several mechanisms have been proposed to explain the antibacterial mechanism of CDs. For example, physical and mechanical damage, ROS induced oxidative stress, photodynamic antibacterial, antibacterial inhibition of bacterial metabolism and so on.

Bacterial cell wall or outer membrane is an important part of bacteria, which is responsible for maintaining cell shape, preventing mechanical stress, infection and osmotic adjustment. When the cell wall or outer membrane of bacteria is physically or mechanically damaged by CDs, the whole physiological function of bacteria is impaired, even the cytoplasm leaks, resulting in the death of bacteria. This is one of the most common antibacterial mechanisms for most types of anti-bacterial CDs. Travlou et al. ${ }^{19}$ Obtained sulfur and nitrogen doped carbon quantum dots (s-cqds and n-cqds) by simple hydrothermal treatment of organic compounds / polymers containing $\mathrm{s}$ or $\mathrm{N}$, and studied their antibacterial activities by Bacillus subtilis and Escherichia coli. Compared with s-cqds, n-cqds had better antibacterial properties.

Functionalized CDs can lead to bacterial death by inhibiting microbial metabolism. Bacteria transform nutrients into energy through metabolism, and eliminate metabolite molecules to inhibit the growth, reproduction and survival of bacteria. Compared with eukaryotic cells, bacteria lack mitochondria, so they are involved in energy metabolism related reactions, which are carried out by ATPase located in the cell membrane. Peptidoglycan is an important component of bacterial cell wall, which can protect bacteria from damage. D-glutamic acid (s-glu) is the precursor of peptidoglycan (PG). Therefore, the inhibition of d-glu-related catalytic enzymes and the disruption of PG biosynthesis can be used to eliminate bacteria and maintain the integrity of mammalian cells. Xin et al. ${ }^{20}$ Used D-Glu functionalized GQD (DGG) to penetrate bacterial cell membrane and target murd ligase to inhibit its catalytic activity in intracellular PG biosynthesis, resulting in cell wall damage, cytoplasmic leakage and cell death. So as to achieve effective antibacterial activity and high selective toxicity.

Jian et al. ${ }^{21}$ used polyamines (including spermine, spermidine and putrescine) as substrates to prepare CQDS with blue fluorescence and positive charge by 
hydrothermal method, and the average particle size was 6 $\mathrm{nm}$. The CQDS prepared with Spermidine as substrate had high quantum yield, and had high toxicity to Escherichia coli, Staphylococcus aureus, Pseudomonas aeruginosa, Salmonella enteritidis and methicillin-resistant Staphylococcus aureus Cocci have good antibacterial activity. Stankovic et al. ${ }^{22}$ synthesized hydrophobic carbon quantum dots (CQDS) by hydrothermal method using polyoxyethylene / polyoxypropylene / polyoxyethylene block copolymer as substrate and deposited on LB films. Under blue light irradiation at 470nm wavelength, they can produce singlet oxygen, which has antibacterial activity against Escherichia coli and Staphylococcus aureus.

\section{Conclusion}

Compared with traditional antibiotics or biological antibacterial agents, nano enzyme as a new antibacterial agent has many advantages; The enzyme activity can be adjusted and optimized by controlling the nano scale factors; Nano enzymes can work together to enhance the antibacterial effect; The combination of functionalized modification and various nano effects can further enhance its bactericidal ability.

However, the research on antibacterial application of nano enzyme is just beginning, and its related mechanism and application need to be further explored. On the one hand, the antibacterial effect of nanozyme against Escherichia coli, Staphylococcus aureus, Pseudomonas aeruginosa, Streptococcus mutans and other pathogenic bacteria is unclear; On the other hand, the specificity of nano enzyme sterilization needs to be explored. Antibiotic sterilization has a specific antibacterial spectrum, The reason is that antibiotics can inhibit the activity of protein or enzyme in specific bacteria. The target of ROS attack mediated by nano enzyme is random and the selectivity is poor. Whether the specific sterilization of nanoenzyme can be achieved by modification or optimization needs further development and exploration.

As a new antibacterial carrier material, carbon based nanozyme has obvious advantages over other traditional antibacterial materials, such as easy preparation, rapid reaction, low cost, high stability, and no bacterial resistance. However, the broad-spectrum and selectivity of nano enzyme antibacterial agents still need to be improved. Through compounding with other antibacterial materials, its sterilization and disinfection performance can be greatly improved, so as to obtain stable, broad-spectrum and green antibacterial agents, which will be the main development trend in the future.

\section{References}

1. IIJIMA, Sumio. Helical microtubules of graphitic carbon[J].nature, 1991, 354.6348: 56.

2. KANG, Seoktae, et al. Single-walled carbon nanotubes exhibit strong antimicrobial activity[J]. Langmuir, 2007, 23.17: 8670-8673.

3. YANG, Cheenou, et al. Antimicrobial activity of single-walled carbon nanotubes: length effect[J]. Langmuir, 2010, 26.20: 16013-16019.

4. LIU, Shaobin, et al. Sharper and faster "nano darts" kill more bacteria: a study of antibacterial activity of individually dispersed pristine single-walled carbon nanotube[J].ACS nano, 2009, 3.12: 3891-3902.

5. ARIAS, L. Renea; YANG, Liju. Inactivation of bacterial pathogens by carbon nanotubes in suspensions[J]. Langmuir, 2009, 25.5: 3003-3012.

6. Liu T, Tang H Q, Cai X M, et al. A study on bactericidal properties of $\mathrm{Ag}$ coated carbon nanotubes[J].Nuclear Instruments and Methods in Physics Research B,2007,264:282-286.

7. ZARDINI, Hadi Zare, et al. Enhanced antibacterial activity of amino acids-functionalized multi walled carbon nanotubes by a simple method[J].Colloids and Surfaces B: Biointerfaces, 2012, 92: 196-202.

8. ASLAN, Seyma, et al. Carbon nanotube-based antimicrobial biomaterials formed via layer-by-layer assembly with polypeptides[J].Journal of colloid and interface science, 2012, 388.1: 268-273.

9. DONG, Xiuli, et al. Inhibitory effects of nisin-coated multi-walled carbon nanotube sheet on biofilm formation from Bacillus anthracis spores[J].Journal of Environmental Sciences, 2014, 26.12: 2526-2534.

10. HU, Wenbing, et al. Graphene-based antibacterial paper[J].ACS nano, 2010, 4.7: 4317-4323.

11. LIU, Shaobin, et al. Antibacterial activity of graphite, graphite oxide, graphene oxide, and reduced graphene oxide: membrane and oxidative stress. ACS nano, 2011, 5.9: 6971-6980.

12. LIU, Lei, et al. Facile synthesis of monodispersed silver nanoparticles on graphene oxide sheets with enhanced antibacterial activity. New Journal of Chemistry, 2011, 35.7: 1418-1423.

13. Lim H N, Huang N M, Loo C H. Facile preparation of grapheme -based chitosan films: Enhanced thermal mechanical and antibacterial properties[J]. Joural of NonCrystalline Solids, 2012 (358): 525-530.

14. Sun H J, Gao N, Dong K, et al. Graphene quantum dots-band-aids used for wound disinfection. Acs Nano, 2014, 8(6): 6202-6210 .

15. Wang Z, Dong K, Liu Z, et al. Activation of biologically relevant levels of reactive oxygen species by $\mathrm{Au} / \mathrm{g}-\mathrm{C} 3 \mathrm{~N} 4$ hybrid nanozyme for bacteria killing and wound disinfection. Biomaterials, 2017,113: 145157

16. Tao $\mathrm{Y}, \mathrm{Ju}$ E G, Ren J S, et al. Bifunctionalized mesoporous silica-supported gold nanoparticles: intrinsic oxidase and peroxidase catalytic activities for antibacterial applications. Adv Mater, 2015, 27(6): 1097-1104.

17. Ji H, Dong K, Yan Z, et al. Bacterial hyaluronidase self-triggered prodrug release for chemophotothermal synergistic treatment of bacterial infection. Small, 2016, 12(45): 6200-6206 
18. RIGODANZA, Francesco, et al. Customizing the Electrochemical Properties of Carbon Nanodots by Using Quinones in Bottom - Up Synthesis. Angewandte Chemie, 2018, 130.18: 5156-5161.

19. Travlou N A, Giannakoudakis D A, Algarra M, et al. S-and N-doped carbon quantum dots: Surface chemistry dependent antibacterial activity $[\mathrm{J}]$. Carbon, 2018, 135:104-111.

20. Xin Q, Liu Q, Geng L, et al. Chiral nanoparticle as a new efficient antimicrobial nanoagent[J].Advanced healthcare materials, 2017, 6(4): 1601011.

21. JIAN, Hong-Jyuan, et al. Super-cationic carbon quantum dots synthesized from spermidine as an eye drop formulation for topical treatment of bacterial keratitis. ACS nano, 2017, 11.7: 6703-6716.

22. STANKOVIĆ, Nenad K., et al. Antibacterial and antibiofouling properties of light triggered fluorescent hydrophobic carbon quantum dots Langmuir-Blodgett thin films. ACS Sustainable Chemistry \& Engineering, 2018, 6.3: 4154-4163. 